\title{
ON THE GENERALIZED POTENTIAL PROBLEM FOR A SURFACE OF REVOLUTION
}

\author{
V. I. FABRIKANT, T. S. SANKAR AND M. N. S. SWAMY
}

\begin{abstract}
The following generalized potential problem is considered: given an arbitrary potential distribution on a surface of revolution, the charge density is to be determined. The problem is called generalized due to the assumption that the point charge potential diminishes with the distance $R$ as $R^{-1}{ }^{\prime \prime},|\nu|<1$. The particular case of $\nu=0$ corresponds to the electrostatic potential problem. A closed form solution to the problem is obtained for a certain class of surfaces of revolution due to a special integral representation of the kernel of the governing integral equation. Three examples are considered: the uniform potential distribution over a spherical cap, the case of an earthed conducting spherical cap in a uniform external field acting in two different directions, namely along the $0 z$ axis and the $0 x$ axis, respectively. The expressions for the charge density distributions are determined for each of the examples. The general results presented in this paper may also be applied to the solution of the mathematically identitical problems in hydrodynamics, thermal conductivity, etc.
\end{abstract}

1. Introduction. The classical electrostatic potential problem has attracted the attention of scientists since 1850 . The earliest published results were related to the axisymmetrical problems. A list of references, together with some applications of the results to certain problems in hydrodynamics, can be found in [1]. The latest published result is believed to be [2], where the exact solution in the form of Fourier series was given to an asymmetric electrostatic problem for a spherical cap. A certain generalization of the electrostatic potential problem is considered in this paper. An assumption is introduced: that the point charge potential diminishes with the distance $R$, as $R^{-1-\nu},|\nu|<1$. The potential problem, related to this assumption, is called hereafter the generalized potential problem. One of its immediate applications, with all the related references, may be found in [3].

The problem formulation and its exact solution is given in the next section. Three examples are considered in $\S 3$; some general remarks may be found in $\S 4$.

2. Formulation of the problem and its solution. Consider an arbitrary surface of revolution, the axis $0 z$ being the axis of revolution. Introduce a set of orthogonal curvilinear coordinates $u$ and $v$, with their relations to the cartesian set as

$$
x=f(u) \cos v, \quad y=f(u) \sin v, \quad z=w(u) .
$$

Received by the editors December 8, 1982.

1980 Mathematics Subject Classification. Primary 31B20, 31B10: Secondary 45A05, 45E10.

1984 Amcrican Mathematical Society $0002-9939 / 84 \$ 1.00+\$ .25$ per page 
Here the functions $f$ and $w$ are known and are defined by the form of the surface of revolution. The distance $R$ between any two points $(u, v)$ and $\left(u_{0}, v_{0}\right)$ on the surface may be defined as

(2)

$$
\begin{aligned}
& R^{2}=\left[f(u) \cos v-f\left(u_{0}\right) \cos v_{0}\right]^{2}+\left[f(u) \sin v-f\left(u_{0}\right) \sin v_{0}\right]^{2}+\left[w(u)-w\left(u_{0}\right)\right]^{2} \\
& \quad=h_{1}\left(u, u_{0}\right)-h\left(u, u_{0}\right) \cos \left(v-v_{0}\right) ; \\
& h\left(u, u_{0}\right)=2 f(u) f\left(u_{0}\right), \quad h_{1}\left(u, u_{0}\right)=f^{2}(u)+f^{2}\left(u_{0}\right)+\left[w(u)-w\left(u_{0}\right)\right]^{2} .
\end{aligned}
$$

Writing

$$
\begin{aligned}
& h_{1}\left(u, u_{0}\right)-h\left(u, u_{0}\right) \cos \left(v-v_{0}\right) \\
& =h\left(u, u_{0}\right) \frac{\left[\xi_{1}\left(u, u_{0}\right)-2 \xi_{1}\left(u, u_{0}\right) \xi_{2}\left(u, u_{0}\right) \cos \left(v-v_{0}\right)+\xi_{2}\left(u, u_{0}\right)\right]}{2}
\end{aligned}
$$

where

$$
\xi_{i}\left(u, u_{0}\right)=\frac{h_{1}\left(u, u_{0}\right)}{h\left(u, u_{0}\right)}+(-1) \sqrt{h_{1}^{2}\left(u, u_{0}\right) / h^{2}\left(u, u_{0}\right)-1} \quad \text { for } i=1,2,
$$

it may be noted that

$$
\xi_{1}\left(u, u_{0}\right)=1 / \xi_{2}\left(u, u_{0}\right)
$$

Using (2), (4) and (5), one establishes also

$$
\xi_{1}(u, u)=\xi_{2}(u, u)=1 \text {. }
$$

Assume the existence of a function $\zeta$ such that

$$
\xi_{1}\left(u, u_{0}\right)=\zeta(u) / \zeta\left(u_{0}\right), \quad \xi_{2}\left(u, u_{0}\right)=\zeta\left(u_{0}\right) / \zeta(u) .
$$

Later it will be shown how to find such a function for certain systems of coordinates. Substitution of (7) and (3) into (2) leads to the relationship

$$
R^{2}=\frac{f(u) f\left(u_{0}\right)}{\zeta(u) \zeta\left(u_{0}\right)}\left[\zeta^{2}(u)-2 \zeta(u) \zeta\left(u_{0}\right) \cos \left(v-v_{0}\right)+\zeta^{2}\left(u_{0}\right)\right]
$$

Now reformulating the problem as: given an arbitrary potential distribution $V(u, v)$ on the surface of revolution $(a \leqslant u \leqslant b, 0 \leqslant v<2 \pi)$, the generalized charge distribution is to be determined. Using (8), one may write the governing integral equation in the form

$$
\begin{array}{r}
\int_{0}^{2 \pi} \int_{u}^{h} \frac{\sigma\left(u_{0}, v_{0}\right) g\left(u_{0}\right) d u_{0} d v_{0}}{\left\{f(u) f\left(u_{0}\right) / \zeta(u) \zeta\left(u_{0}\right)\left[\zeta^{2}(u)-2 \zeta(u) \zeta\left(u_{0}\right) \cos \left(v-v_{0}\right)+\zeta^{2}\left(u_{0}\right)\right]\right\}^{(1+v) / 2}} \\
=V(u, v) \text { for } a \leqslant u \leqslant b, 0 \leqslant v<2 \pi .
\end{array}
$$

For the system of curvilinear coordinates (1), the Jacobian $g$ is given by

$$
g(u)=f(u) \sqrt{(d f(u) / d u)^{2}+(d w(u) / d u)^{2}} .
$$


Making use of the integral representation established in [3],

(11)

$$
\begin{aligned}
& \frac{1}{\left[r^{2}-2 r r_{0} \cos \left(v-v_{0}\right)+r_{0}^{2}\right]^{(1+\nu) / 2}} \\
& =\frac{2}{\pi} \cos \frac{\pi \nu}{2} \int_{0}^{\min \left(r_{0} r_{0}\right)} \frac{x^{\nu} d x}{\left[\left(r^{2}-x^{2}\right)\left(r_{0}^{2}-x^{2}\right)\right]^{(1+\nu) / 2}} \\
& \quad \times\left[\frac{1}{1-x^{2} e^{-i\left(v-v_{0}\right)} / r r_{0}}+\frac{1}{1-x^{2} e^{i\left(v-v_{0}\right)} / r r_{0}}-1\right] .
\end{aligned}
$$

Assuming $r \equiv \zeta(u), r_{0} \equiv \zeta\left(u_{0}\right)$ and $x \equiv \zeta(t)$, one will have, instead of (11),

$$
\begin{aligned}
& \frac{1}{\left[\zeta^{2}(u)-2 \zeta(u) \zeta\left(u_{0}\right) \cos \left(v-v_{0}\right)+\zeta^{2}\left(u_{0}\right)\right]^{(1+v) / 2}} \\
& =\frac{2}{\pi} \cos \frac{\pi \nu}{2} \int_{c}^{\min \left(u, u_{0}\right)} \frac{\chi\left(t, u, u_{0}, v, v_{0}\right) \zeta^{\nu}(t) d \zeta(t)}{\left\{\left[\zeta^{2}(u)-\zeta^{2}(t)\right]\left[\zeta^{2}\left(u_{0}\right)-\zeta^{2}(t)\right]\right\}^{(1+\nu) / 2}} .
\end{aligned}
$$

Here, $c=\zeta^{-1}(0)$,

$$
\begin{aligned}
& \chi(\left.t, u, u_{0}, v, v_{0}\right) \\
& \quad=\left[\frac{1}{1-\zeta^{2}(t) e^{-i\left(v-v_{0}\right)} / \zeta(u) \zeta\left(u_{0}\right)}+\frac{1}{1-\zeta^{2}(t) e^{i\left(v-v_{0}\right)} / \zeta(u) \zeta\left(u_{0}\right)}-1\right]
\end{aligned}
$$

and $\zeta^{-1}$ denotes the function inverse of $\zeta$. Also, let

$$
\zeta^{-1}(0)=a
$$

Later it will be shown that this requirement is not so limiting. Now substitution of (14) and (12) into (9) leads to the governing integral equation

$$
\begin{array}{r}
\frac{2}{\pi} \cos \frac{\pi \nu}{2} \int_{a}^{u} \frac{\zeta^{\nu}(t) d \zeta(t)}{\left[\zeta^{2}(u)-\zeta^{2}(t)\right]^{(1+\nu) / 2}} \int_{t}^{h} \frac{G\left(u_{0}\right) d u_{0}}{\left[\zeta^{2}\left(u_{0}\right)-\zeta^{2}(t)\right]^{(1+\nu) / 2}} \\
\quad \times \int_{0}^{2 \pi} \chi\left(t, u, u_{0}, v, v_{0}\right) \sigma\left(u_{0}, v_{0}\right) d v_{0}=\Omega(u, v)
\end{array}
$$

Here

$$
G\left(u_{0}\right)=\left[\zeta\left(u_{0}\right) / f\left(u_{0}\right)\right]^{(1+\nu) / 2} g\left(u_{0}\right), \quad \Omega(u, v)=[f(u) / \zeta(u)]^{(1+\nu) / 2} V(u, v) .
$$

The following scheme of change of the order of integration was employed:

$$
\begin{aligned}
\int_{a}^{b} d u_{0} \int_{a}^{\min \left(u, u_{0}\right)} d t & =\int_{a}^{u} d u_{0} \int_{a}^{u_{0}} d t+\int_{u}^{b} d u_{0} \int_{a}^{u} d t \\
& =\int_{a}^{u} d t \int_{t}^{u} d u_{0}+\int_{a}^{u} d t \int_{u}^{b} d u_{0}=\int_{a}^{u} d t \int_{t}^{h} d u_{0} .
\end{aligned}
$$


Equation (15), despite looking more complicated than the original equation (9), admits exact solution in a closed form, using a technique developed in [3]. Introduce the operator

$$
L(k) \phi(u, v)=2 \pi \sum_{n=-\infty}^{\infty} k^{|n|} a_{n}(u) e^{i n c}, \quad 0 \leqslant k<\infty,
$$

where

$$
a_{n}(u)=\frac{1}{2 \pi} \int_{0}^{2 \pi} \phi(u, v) e^{-i n t} d v
$$

The following properties of the $L$-operator were established in [3]

$$
\begin{aligned}
& L(k) L\left(k_{1}\right)=2 \pi L\left(k k_{1}\right), \\
& L(1) \phi(u, v)=2 \pi \phi(u, v) .
\end{aligned}
$$

These properties allow one to construct the inverse operator

$$
L^{-1}(k)=\frac{1}{2 \pi} L\left(\frac{1}{k}\right) .
$$

Equation (15) may be rewritten by introducing the $L$-operators

$$
\begin{array}{r}
\frac{2}{\pi} \cos \frac{\pi \nu}{2} \int_{a}^{u} \frac{\zeta^{\nu}(t) d \zeta(t)}{\left[\zeta^{2}(u)-\zeta^{2}(t)\right]^{(1+\nu) / 2}} \int_{t}^{b} \frac{G\left(u_{0}\right) d u_{0}}{\left[\zeta^{2}\left(u_{0}\right)-\zeta^{2}(t)\right]^{(1+v) / 2}} L\left(\frac{\zeta^{2}(t)}{\zeta^{2}(u) \zeta^{2}\left(u_{0}\right)}\right) \\
\times \sigma\left(u_{0}, v\right)=\Omega(u, v) \text { for } a \leqslant u \leqslant b, 0 \leqslant v<2 \pi .
\end{array}
$$

Now the governing equation (21) presents a sequence of two integral operators of Abel type and the $L$-operator. The inverse operator to each one is known. An exact solution of (21) may now be constructed in several steps. Convergency at each step is not verified, but is assumed. Application of the operator

$$
\frac{d}{d u_{1}} \int_{a}^{u_{1}} \frac{\zeta(u) d \zeta(u)}{\left[\zeta^{2}\left(u_{1}\right)-\zeta^{2}(u)\right]^{(1-\nu) / 2}} L(\zeta(u))
$$

to both parts of $(21)$ gives

$$
\begin{gathered}
2 \pi \zeta^{\prime}\left(u_{1}\right) \zeta^{\nu}\left(u_{1}\right) \int_{u_{1}}^{h} \frac{G\left(u_{0}\right) d u_{0}}{\left[\zeta^{2}\left(u_{0}\right)-\zeta^{2}\left(u_{1}\right)\right]^{(1+\nu) / 2}} L\left(\frac{\zeta^{2}\left(u_{1}\right)}{\zeta\left(u_{0}\right)}\right) \sigma\left(u_{0}, v\right) \\
=\frac{d}{d u_{1}} \int_{a}^{u_{1}} \frac{\zeta(u) d \zeta(u)}{\left[\zeta^{2}\left(u_{1}\right)-\zeta^{2}(u)\right]^{(1-v) / 2}} L(\zeta(u)) \Omega(u, v)
\end{gathered}
$$

Hereafter, the properties of $L$-operators (18) and (19) are employed; a prime indicates the derivative with respect to the variable given in brackets. The following integral [5] is also used. 
THE (;ENERALIZED POTENTIAL PROBLEM

51

$$
\int_{t}^{u_{1}} \frac{\zeta(u) d \zeta(u)}{\left[\zeta^{2}\left(u_{1}\right)-\zeta^{2}(u)\right]^{(1-\nu) / 2}\left[\zeta^{2}(u)-\zeta^{2}(t)\right]^{(1+\nu) / 2}}=\frac{\pi}{2 \cos (\pi \nu / 2)}
$$

The next step is the application of the operator

$$
\frac{d}{d u_{2}} \int_{u_{2}}^{b} \frac{\zeta^{1-\nu}\left(u_{1}\right) d u_{1}}{\left[\zeta^{2}\left(u_{1}\right)-\zeta^{2}\left(u_{2}\right)\right]^{(1-\nu) / 2}} L\left(\frac{1}{\zeta^{2}\left(u_{1}\right)}\right)
$$

to both parts of equation (22). The result then is

$$
\begin{aligned}
-\frac{2 \pi^{3}}{\cos (\pi \nu / 2)} & G\left(u_{2}\right) L\left(\frac{1}{\zeta\left(u_{2}\right)}\right) \sigma\left(u_{2}, v\right) \\
= & \frac{d}{d u_{2}} \int_{u_{2}}^{h} \frac{\zeta^{1-\nu}\left(u_{1}\right) d u_{1}}{\left[\zeta^{2}\left(u_{1}\right)-\zeta^{2}\left(u_{2}\right)\right]^{(1-\nu) / 2}} L\left(\frac{1}{\zeta^{2}\left(u_{1}\right)}\right) \\
& \times \frac{d}{d u_{1}} \int_{a}^{u_{1}} \frac{\zeta(u) d \zeta(u)}{\left[\zeta^{2}\left(u_{1}\right)-\zeta^{2}(u)\right]^{(1-\nu) / 2}} L(\zeta(u)) \Omega(u, v) .
\end{aligned}
$$

Applying the operator $L\left(\zeta\left(u_{2}\right)\right)$ to both parts of (24) and using the property (20), one finally gets the solution

$$
\begin{aligned}
\sigma\left(u_{2}, v\right)= & -\frac{\cos (\pi \nu / 2)}{8 \pi^{5} G\left(u_{2}\right)} L\left(\zeta\left(u_{2}\right)\right) \frac{d}{d u_{2}} \int_{u_{2}}^{h} \frac{\zeta^{1-\nu}\left(u_{1}\right) d u_{1}}{\left[\zeta^{2}\left(u_{1}\right)-\zeta^{2}\left(u_{2}\right)\right]^{(1-\nu) / 2}} \\
& \times L\left(\frac{1}{\zeta^{2}\left(u_{1}\right)}\right) \frac{d}{d u_{1}} \int_{a}^{u_{1}} \frac{\zeta(u) d \zeta(u)}{\left[\zeta^{2}\left(u_{1}\right)-\zeta^{2}(u)\right]^{(1-\nu) / 2}} L(\zeta(u)) \Omega(u, v) .
\end{aligned}
$$

One may establish the following rules of differentiation under the integral sign:

$$
\text { (26) } \begin{aligned}
& \frac{d}{d u_{1}} \int_{a}^{u_{1}} \frac{\phi(u) \zeta(u) d \zeta(u)}{\left[\zeta^{2}\left(u_{1}\right)-\zeta^{2}(u)\right]^{(1-\nu) / 2}}\left\{\frac{\phi(a)}{\left[\zeta^{2}\left(u_{1}\right)-\zeta^{2}(a)\right]^{(1-\nu) / 2}}+\int_{a}^{u_{1}} \frac{d \phi(u)}{\left[\zeta^{2}\left(u_{1}\right)-\zeta(u)\right]^{(1-\nu) / 2}}\right\} \zeta\left(u_{1}\right) \zeta^{\prime}\left(u_{1}\right), \\
& \frac{d}{d u_{2}} \int_{u_{2}}^{b} \frac{\phi\left(u_{1}\right) \zeta\left(u_{1}\right) d \zeta\left(u_{1}\right)}{\left[\zeta^{2}\left(u_{1}\right)-\zeta^{2}\left(u_{2}\right)\right]^{(1-\nu) / 2}} \\
&=\left\{-\frac{\phi(b)}{\left[\zeta^{2}(b)-\zeta^{2}\left(u_{2}\right)\right]^{(1-\nu) / 2}}+\int_{u_{2}}^{b} \frac{d \phi\left(u_{1}\right)}{\left[\zeta^{2}\left(u_{1}\right)-\zeta^{2}\left(u_{2}\right)\right]^{(1-\nu) / 2}}\right\} \zeta\left(u_{2}\right) \zeta^{\prime}\left(u_{2}\right) .
\end{aligned}
$$


Further simplification of (25) is possible by using (26) and the properties of the $L$-operator (18), (19). The result then is

$$
\begin{array}{r}
\sigma\left(u_{2}, v\right)=\frac{\cos (\pi \nu / 2)}{2 \pi^{3} G\left(u_{2}\right)} \zeta\left(u_{2}\right) \zeta^{\prime}\left(u_{2}\right)\left\{\frac{\Phi\left(b, u_{2}, v\right)}{\left[\zeta^{2}(b)-\zeta^{2}\left(u_{2}\right)\right]^{(1-v) / 2}}\right. \\
\left.-\int_{u_{2}}^{l} \frac{d \Phi\left(u_{1}, u_{2}, v\right) / d u_{1}}{\left[\zeta^{2}\left(u_{1}\right)-\zeta^{2}\left(u_{2}\right)\right]^{(1-v) / 2}} d u_{1}\right\}
\end{array}
$$

$\Phi\left(u_{1}, u_{2}, v\right)$

$$
\begin{aligned}
=\zeta^{\prime} v^{\prime}\left(u_{1}\right)\left\{\frac{\int_{0}^{2 \pi} \Omega(a, v) d v}{\left[\zeta^{2}\left(u_{1}\right)-\zeta^{2}(a)\right]^{(1-v) / 2}}\right. \\
\left.\quad+\int_{a}^{u_{1}} \frac{d u}{\left[\zeta^{2}\left(u_{1}\right)-\zeta^{2}(u)\right]^{(1-v) / 2}} \frac{d}{d u}\left[L\left(\frac{\zeta(u) \zeta\left(u_{2}\right)}{\zeta^{2}\left(u_{1}\right)}\right) \Omega(u, v)\right]\right\} .
\end{aligned}
$$

The solution of the problem is now complete; expressions (25) and (27) give the two different forms of presentation of the solution.

3. Examples. Consider a spherical cap as an example of a surface of revolution. If the cap radius is $r$, then

$$
f(u)=r \sin u, \quad w(u)=r \cos u, \quad a=0
$$

and formulae (2), (4) and (7) give

$$
\xi_{1}\left(u, u_{0}\right)=\frac{\tan (u / 2)}{\tan \left(u_{0} / 2\right)}=\frac{1}{\xi_{2}\left(u, u_{0}\right)}, \quad \zeta(u)=\tan \frac{u}{2} .
$$

Since $\zeta^{-1}(u)=2 \tan ^{-1} u$, the requirement in (14) is satisfied and the solution (27) is valid.

The simplest case of a charged spherical cap is the one of uniform potential over the surface, namely $V(u, v)=V_{0}=$ const; and a consecutive application of the formulae (16), (29) and (27) gives

$$
\begin{gathered}
\Omega(u, v)=V_{0}(2 r)^{\left(1+\nu^{\prime}\right) / 2} \cos ^{1+\nu} \frac{u}{2}, \quad G\left(u_{0}\right)=r(2 r)^{(1-\nu) / 2} \sin \frac{u}{2} \cos ^{-\nu^{\prime}} \frac{u}{2}, \\
\Phi\left(u_{1}, u_{2}, v\right)=2 \pi V_{0}(2 r)^{(1+\nu) / 2} \cos ^{2} \frac{u_{1}}{2} \\
\quad+\frac{V_{0} \cos (\pi \nu / 2)}{(2 r)^{1-\nu} \pi^{2}}\left\{\frac{1-\nu}{1+\nu} z^{(1+\nu) / 2} F\left(\frac{1+\nu}{2}, \frac{1+\nu}{2}, \frac{3+\nu}{2} ; z\right)\right. \\
{\left[\cos ^{2}\left(u_{2} / 2\right)-\cos ^{2}(b / 2)\right]^{(1-\nu) / 2}(b / 2)} \\
z=1-\frac{\cos ^{2}(b / 2)}{\cos ^{2}\left(u_{2} / 2\right)}
\end{gathered}
$$


Here $F(\alpha, \beta ; \gamma ; z)$ is the Gauss hypergeometric function, and the following integral was employed [5].

$$
\begin{aligned}
\int_{u_{2}}^{b} \frac{\cos ^{\beta} \frac{u_{1}}{2} d\left(\cos \left(u_{1} / 2\right)\right)}{\left(\cos ^{2} \frac{u_{2}}{2}-\cos ^{2} \frac{u_{1}}{2}\right)^{(1-\nu) / 2}}= & -\frac{\cos ^{\beta-1}\left(u_{2} / 2\right)}{1+\nu}\left(\cos ^{2} \frac{u_{2}}{2}-\cos ^{2} \frac{b}{2}\right)^{(1+\nu) / 2} \\
& \times F\left(\frac{1-\beta}{2}, \frac{1+\nu}{2} ; \frac{3+\nu}{2} ; z\right) .
\end{aligned}
$$

The classical case of the electrostatic potential problem corresponds to $\nu=0$, and the result (30) may be expressed in terms of elementary functions

$$
\sigma\left(u_{2}, v_{3}\right)=\frac{V_{0}}{2 \pi^{2} r}\left[\cos ^{-1} \frac{\cos (b / 2)}{\cos \left(u_{2} / 2\right)}+\frac{\cos (b / 2)}{\left(\cos ^{2}\left(u_{2} / 2\right)-\cos ^{2}(b / 2)\right)^{1 / 2}}\right] .
$$

This is in accordance with the known result [1]. Here the following property of the hypergeometric function was used [5].

$$
\sqrt{z} F\left(\frac{1}{2}, \frac{1}{2} ; \frac{3}{2} ; z\right)=\sin ^{-1} \sqrt{z}
$$

As a second example, consider the case of an earthed spherical cap in a uniform electric field of intensity $E$, acting along the $0 z$ axis. For this case, $V(u, v)=$ Er cos $u$ and formulae (16) and (27) give

$$
\begin{gathered}
\Omega(u, v)=\operatorname{Er}(2 r)^{(1+\nu) / 2} \cos ^{1+\nu} \frac{u}{2} \cos u, \quad G(u)=r(2 r)^{(1-\nu) / 2} \sin \frac{u}{2} \cos ^{-\nu} \frac{u}{2}, \\
\Phi\left(u_{1}, u_{2}, v\right)=2 \pi E r(2 r)^{(1+\nu) / 2} \cos ^{2} \frac{u}{2}\left(4 \cos ^{2} \frac{u}{2}-3+\nu\right) /(1+\nu) . \\
\sigma\left(u_{2}, v\right)=\frac{E r^{\nu} \cos (\pi \nu / 2)}{2^{1-\nu}(1+\nu) \pi^{2}} \\
\quad \times\left\{\left(\cos ^{1-\nu} \frac{b}{2}\right) \frac{(3-\nu) \cos u_{2}-(1-\nu)(1+\cos b)}{\left(\cos ^{2}\left(u_{2} / 2\right)-\cos ^{2}(b / 2)\right)^{(1-\nu) / 2}}\right. \\
\left.\quad+\frac{(3-\nu)(1-\nu)}{1+\nu} z^{(1+\nu) / 2} \cos u_{2} F\left(\frac{1+\nu}{2}, \frac{1+\nu}{2} ; \frac{3+\nu}{2} ; z\right)\right\}, \\
z=1-\frac{\cos ^{2}(b / 2)}{\cos ^{2}\left(u_{2} / 2\right)} .
\end{gathered}
$$


Here integral (31) was employed, as well as the following properties of the hypergeometric functions [5].

$$
\text { 34) } \begin{aligned}
F\left(-\frac{1-\nu}{2},\right. & \left.\frac{1+\nu}{2} ; \frac{3+\nu}{2} ; z\right) \\
= & \frac{1+\nu}{2}(1-z)^{(1-\nu) / 2}+\frac{1-\nu}{2} F\left(\frac{1+\nu}{2}, \frac{1+\nu}{2} ; \frac{3+\nu}{2} ; z\right), \\
F\left(-\frac{3-\nu}{2}, \frac{1+\nu}{2} ; \frac{3+\nu}{2} ; z\right)= & \frac{(1-\nu)(3-\nu)}{8} F\left(\frac{1+\nu}{2}, \frac{1+\nu}{2} ; \frac{3+\nu}{2} ; z\right) \\
& -\frac{1+\nu}{8}(2 z-5+\nu)(1-z)^{(1-\nu) / 2},
\end{aligned}
$$

$$
F(d, \beta ; \beta ; z)=(1-z)^{-d} \text {. }
$$

Result (33) may be simplified for the particular case $\nu=0$, and it may be expressed in elementary functions

$\sigma\left(u_{2}, v\right)=\frac{E}{2 \pi^{2}}\left[\left(\cos \frac{b}{2}\right) \frac{3 \cos u_{2}-1-\cos b}{\sqrt{\cos ^{2}\left(u_{2} / 2\right)-\cos ^{2}(b / 2)}}+3 \cos u_{2} \cos ^{-1}\left(\frac{\cos (b / 2)}{\cos \left(u_{2} / 2\right)}\right)\right]$.

The following relationships, established by the usage of (32) and (34), were employed:

$$
\begin{gathered}
F\left(-\frac{1}{2}, \frac{1}{2} ; \frac{3}{2} ; z\right)=\frac{\sin ^{-1} \bar{z}}{2 \bar{z}}+\frac{1 \overline{1-z}}{2}, \\
F\left(-\frac{3}{2}, \frac{1}{2} ; \frac{3}{2} ; z\right)=\frac{3 \sin ^{-1} \bar{z}}{8 \bar{z}}+\frac{5-2 z}{8}, \overline{1-z} .
\end{gathered}
$$

The two examples considered were axisymmetric. Now consider an asymmetric problem, the case of an earthed spherical cap in a uniform electric field, acting in the $0 x$ direction. For this case $V(u, v)=E r \sin u \cos v$, and the result of the use of (16) and (27) is

$$
\begin{gathered}
\Omega(u, v)=E r(2 r)^{(1+\nu) / 2} \cos ^{1+\nu} \frac{u}{2} \sin u \cos v, \\
G(u)=r(2 r)^{(1-\nu) / 2} \sin \frac{u}{2} \cos ^{-\nu} \frac{u}{2} \\
\Phi\left(u_{1}, u_{2}, v\right)=\frac{8 \pi E r}{1+\nu}(2 r)^{\left(1+\nu^{\prime}\right) / 2} \cos v \tan \frac{u_{2}}{2} \cos ^{4} \frac{u_{1}}{2}, \\
\sigma\left(u_{2}, v\right)=\frac{2 E(2 r)^{\nu} \cos (\pi \nu / 2)}{\pi^{2}(1+\nu)} \cos v \tan \frac{u_{2}}{2} \\
\times\left\{\frac{3-\nu}{1+\nu} \cos ^{2} \frac{u_{2}}{2} z^{(1+\nu) / 2} F\left(-\frac{1-\nu}{2}, \frac{1+\nu}{2} ; \frac{3+\nu}{2} ; z\right)\right. \\
z=1-\frac{\cos ^{2}(b / 2)}{\cos ^{2}\left(u_{2} / 2\right)} .
\end{gathered}
$$


Here again, integral (31) and the hypergeometric functions properties (34) were used. When $\nu=0$, the results (36) may be simplified, using (35) as

$$
\begin{aligned}
\sigma\left(u_{2}, v\right)=\frac{E}{\pi^{2}} \cos v \tan \frac{u_{2}}{2}\left\{\left(\cos \frac{b}{2}\right) \frac{3 \cos ^{2}\left(u_{2} / 2\right)-\cos ^{2}(b / 2)}{\sqrt{\cos ^{2}\left(u_{2} / 2\right)-\cos ^{2}(b / 2)}}\right. \\
\left.+3 \cos ^{2} \frac{u_{2}}{2} \cos ^{-1}\left(\frac{\cos (b / 2)}{\cos \left(u_{2} / 2\right)}\right)\right\} .
\end{aligned}
$$

Notice the convenience of the $L$-operator series representation for its evaluation

$$
L(k) \phi(u, v)=\sum_{n=-\infty}^{\infty} k^{|n|} \int_{0}^{2 \pi} e^{i n\left(v-v_{0}\right)} \phi\left(u, v_{0}\right) d v_{0} .
$$

4. Discussion. The method of exact solution to the generalized potential problem, proposed in this paper, may be applied only to those systems of curvilinear coordinates, which satisfy the requirements (7) and (14). This limitation is not that severe, as it might appear. The examples, considered in $\$ 3$, were related to the case of a spherical cap, but this does not mean that the system of the spherical coordinates is the only one to satisfy conditions (7) and (14). For example, the system of polar coordinates on a circular disk also satisfies (7) and (14); namely, for that case $f(u)=u, w(u)=0, a=0, \xi_{1}\left(u, u_{0}\right)=1 / \xi_{2}\left(u, u_{0}\right)=u / u_{0}, \zeta(u)=u$ and formula (27) gives the solution of the generalized potential problem for a circular disk, corresponding to the one previously obtained in [3].

When the surface of revolution is such that the system of curvilinear coordinates does not satisfy the requirements (7) and (14), the procedure of approximation of (4) in the form (7) may be recommended. If the procedure is successful, formula (27) will give an approximate solution to the problem. As an example of such an approximation, consider the case of a cylinder of radius $R$ and height $H$. In this case $f(u)=R, w(u)=u$ and formulae (2) and (4) give

$$
\xi_{1.2}\left(u, u_{0}\right)=1+\frac{1}{2}\left(\frac{u-u_{0}}{R}\right)^{2} \pm\left(\frac{u-u_{0}}{R}\right) \sqrt{1+\left(\frac{u-u_{0}}{2 R}\right)^{2}}
$$

If the condition $(H / R) \ll 1$ holds, the following approximation of (37) is valid:

$$
\xi_{1,2}\left(u, u_{0}\right)=1 \pm\left(\frac{u-u_{0}}{R}\right) .
$$

The last expression may be represented with the same degree of accuracy as

$$
\xi_{1}\left(u, u_{0}\right)=\frac{R+u}{R+u_{0}}, \quad \xi_{2}\left(u, u_{0}\right)=\frac{R+u_{0}}{R+u} .
$$

Now one may assume $\zeta(u)=R+u, a=-R$ and the requirements (7) and (14) are satisfied, then all the results of this paper become applicable to the case of a short cylinder. 
It may also be noticed that it is possible to solve the mathematically identical problems in hydrodynamics, nonhomogeneous elasticity, heat conduction, etc., using the general approach of this paper. The research reported in this paper was supported partially by the grant A7104 from the National Sciences and Research Council of Canada and by the grant N96 from Concordia University.

\section{REFERENCES}

1. W. D. Collins, On the solution of some axisymmetric boundary value problems by means of integral equations, Quart. J. Mech. Appl. Math. 12 (1959), 232-241.

2. D. Homentcovschi, On the electrostatic potential problem for a spherical cap, Z. Angew. Math. Mech. 60 (1980), 636-637.

3. T. S. Sankar and V. I. Fabrikant, Asılmmetric contact problem including wear for nonhomogeneous half-space, J. Appl. Mech. 49 (1982), 43-46.

4. G. A. Korn and T. M. Korn, Mathematical handbook for scientists and engineers, McGraw-Hill, New York, 1968.

5. I. S. Gradshtein and I. M. Ryzhik, Table of integrals, series and products, Academic Press, New York. 1965.

Department of Mechanical Engineering, Concordia University, Montreal, Quebec, Canada H3G 1 M8 (Current address of V. I. Fabrikant and T. S. Sankar)

Department of Electrical Engineering, Concordia University, Montreal, Quebec, Canada H3G 1 M8 (Current address of M. N. S. Swamy) 